\title{
Aerosol deposition in the human lung following administration from a microprocessor controlled pressurised metered dose inhaler
}

\author{
Stephen J Farr, Antony M Rowe, Reid Rubsamen, Glyn Taylor
}

\begin{abstract}
Background - Gamma scintigraphy was employed to assess the deposition of aerosols emitted from a pressurised metered dose inhaler (MDI) contained in a microprocessor controlled device (SmartMist), a system which analyses an inspiratory flow profile and automatically actuates the MDI when predefined conditions of flow rate and cumulative inspired volume coincide.
\end{abstract}

Methods - Micronised salbutamol particles contained in a commercial MDI (Ventolin) were labelled with $99 \mathrm{~m}$-technetium using a method validated by the determination of (1) aerosol size characteristics of the drug and radiotracer following actuation into an eight stage cascade impactor and (2) shot potencies of these non-volatile components as a function of actuation number. Using nine healthy volunteers in a randomised factorial interaction design the effect of inspiratory flow rate (slow, $301 / \mathrm{min}$; medium, $901 / \mathrm{min}$; fast, $270 \mathrm{l} / \mathrm{min}$ ) combined with cumulative inspired volume (early, $300 \mathrm{ml}$; late, $3000 \mathrm{ml}$ ) was determined on total and regional aerosol lung deposition using the technique of gamma scintigraphy.

Results - The SmartMist firing at the medium/early setting (medium flow and early in the cumulative inspired volume) resulted in the highest lung deposition at $18.6(1.42) \%$. The slow/early setting gave the second highest deposition at $14 \cdot 1$ $(2 \cdot 06) \%$ with the fast/late setting resulting in the lowest $(7 \cdot 6(1 \cdot 15) \%)$. Peripheral lung deposition obtained for the medium/early $(9.1(0.9) \%)$ and slow/early (7.5 (1.06)\%) settings were equivalent but higher than those obtained with the other treatments. This reflected the lower total lung deposition at these other settings as no difference in regional deposition, expressed as a volume corrected central zone:peripheral zone ratio, was apparent for all modes of inhalation studied.

Conclusions - The SmartMist device allowed reproducible actuation of an MDI at a preprogrammed point during inspiration. The extent of aerosol deposition in the lung is affected by a change in firing point and is promoted by an inhaled flow rate of up to $901 / \mathrm{min}$ - that is, the slow and medium setting used in these studies. (Thorax 1995;50:639-644)

Keywords: aerosol deposition, metered dose inhaler, microprocessor controlled device, gamma scintigraphy.
There is a growing awareness that optimising the extent of lung deposition (dose) and regional distribution of an inhaled drug could improve its therapeutic efficacy. This has obvious relevance for locally acting drugs such as aerosolised bronchodilators and corticosteroids. In addition, optimised delivery will be essential if the inhaled route is to prove a viable method for delivering systemically active drugs that are poorly absorbed from other sites of administration. For example, differences in regional deposition profoundly affect the bioavailability of macromolecular drugs such as insulin following pulmonary delivery. ${ }^{1}$

SmartMist (Aradigm Corporation, Hayward, California, USA) is a hand held, breath actuated, microprocessor controlled accessory for use with metered dose inhalers (MDIs). Briefly, the device (fig 1) can be loaded with a standard MDI canister to form a highly portable, fully integrated system capable of delivering a drug bolus at a preprogrammed point in the inhalation cycle, defined by both flow rate and cumulative inspiratory volume parameters. Inhalation flow is measured using a pneumotachometer which monitors the inspiratory cycle before, during, and after drug administration. This is recorded in solid state memory for later retrieval via a personal computer.

Gamma camera imaging provides the means for the qualitative and quantitative assessment of the fate of aerosols inhaled from pressurised MDIs. ${ }^{2}$ In this paper a gamma scintigraphic study in a group of healthy subjects is described to compare the extent and pattern of pulmonary deposition of ${ }^{99 \mathrm{~m}} \mathrm{Tc}$-labelled aerosols emitted from a standard commercial MDI fitted within the SmartMist device operating under five different inspiratory conditions of flow and cumulative inspired volume.

\section{Methods}

PREPARATION AND VALIDATION OF RADIOLABELLED MDIS

Salbutamol inhalers, containing $0 \cdot 159 \%$ $\mathrm{w} / \mathrm{v}$ salbutamol in a trichlorofluoromethane (P11)/dichlorodifluoromethane (P12) blend (Ventolin, Allen \& Hanburys, Greenford, UK) were used as a model for suspension type MDIs in the in vivo studies. The radiolabelling method, using $99 \mathrm{~m}$-technetium as pertechnetate, was modified from that first reported by Summers $e t a l^{3}$ for an MDI formulation of nedocromil sodium and which has subsequently been adopted for those containing salbutamol. ${ }^{45}$

The method was validated in two ways. Firstly, the aerosol size distribution of both 


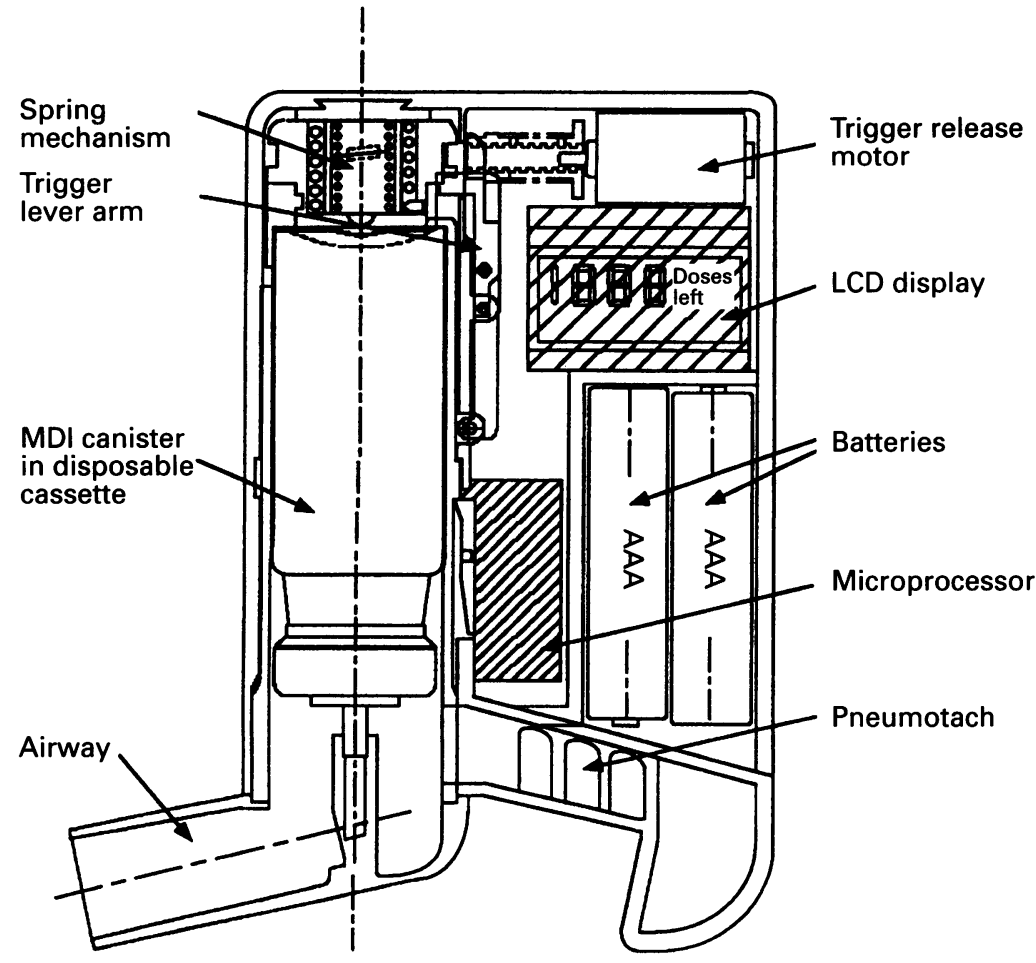

Figure 1 Cut away view of the SmartMist device loaded with a metered dose inhaler (MDI) canister. screening. The subjects were aged between 19 and 26 years and had normal values for forced expiratory volume in one second $\left(\mathrm{FEV}_{1}\right)$ (range 3.69-5.311) and forced vital capacity (FVC) (4.61-6.401) based on predictions calculated from age, weight, and height parameters. All subjects gave written informed consent and the study was approved by the Administration of Radioactive Substances Advisory Committee (ARSAC) and the South Glamorgan Health Authority joint ethics committee.

The SmartMist device was programmed to fire at three different levels of inspiratory flow (slow, medium and fast) and two levels of cumulative inspired volume (early and late) in a randomised, factorial interaction study (table 1). Each volunteer was therefore studied on five occasions with a minimum of 48 hours washout between study phases. This period is equivalent to eight times the half life for ${ }^{99 \mathrm{~m}} \mathrm{Tc}$ decay, although in practice the isotope is rapidly cleared from the lung following aerosol administration (absorption half life 20-30 minutes). ${ }^{7}$

Before taking part each volunteer was required to complete a practice session using the SmartMist system loaded with a nonradioactive, placebo MDI. Thus, each volunteer became competent at inspiring consistently at the low, medium, and high flow rates needed to activate the device. This was assisted by two coloured LEDs located on the device that provided visual feedback to the volunteer for a preprogrammed inspiratory flow rate. No delivery event occurred unless the inspiratory flow and cumulative inspired volume criteria were simultaneously achieved, and no inspiratory flow restriction was introduced to control the subject's inspiratory manoeuvre.

On each study day a primed, radiolabelled MDI was fitted into the preprogrammed SmartMist device which was secured in a customised $0.5 \mathrm{~cm}$ thick lead jacket to shield the volunteer from the high surface dose emitted from the MDI canister containing the radiolabelled formulation. The volunteer, in a seated position, inhaled two puffs from the radiolabelled MDI commencing at residual volume. A five second breath holding manoeuvre after each puff was adopted and the exhaled dose was retrieved on a filter (Whatman GF/A). Immediately after administration posterior and anterior views (200 seconds each) of the lungs and stomach and a lateral head and neck view
STUDY PROTOCOL volunteers selected after appropriate medical

Table 1 SmartMist firing points (inspired flow rate/cumulative inspired volume) set for the various treatments, and mean (SE) inspiratory parameters obtained with the device operating at these firing points in nine volunteers

\begin{tabular}{|c|c|c|c|c|c|}
\hline & \multicolumn{5}{|c|}{ Description of firing point } \\
\hline & Slow/early & Slow/late & Medium/early & Fast/early & Fast/late \\
\hline $\begin{array}{l}\text { Anticipated firing flow }(\mathrm{l} / \mathrm{min}) \\
\text { (set range) } \\
\text { Anticipated firing volume (ml) } \\
\text { (set range) } \\
\text { Actual firing flow (1/min) } \\
\text { Actual firing volume (ml) } \\
\text { Mean flow after dose }(1 / \mathrm{min}) \\
\text { Overall mean flow }(1 / \mathrm{min}) \\
\text { Maximum flow (1/min) } \\
\text { Total inspired volume (TIV) (ml) } \\
\text { TIV/FVC }(\%) \\
\text { Inhalation time (s) }\end{array}$ & $\begin{array}{l}30 \\
(15-45) \\
300 \\
(180-420) \\
30.2(2 \cdot 9) \\
196 \cdot 3(4 \cdot 5) \\
22 \cdot 8(1 \cdot 4) \\
22 \cdot 9(1 \cdot 2) \\
41 \cdot 2(0 \cdot 81) \\
2301(255) \\
44 \cdot 3(4 \cdot 5) \\
6 \cdot 2(0 \cdot 9)\end{array}$ & $\begin{array}{c}30 \\
(15-45) \\
3000 \\
(1800-4200) \\
28 \cdot 2(2 \cdot 1) \\
1868(46) \\
26 \cdot 2(1 \cdot 6) \\
27 \cdot 6(1 \cdot 2) \\
47 \cdot 8(2 \cdot 5) \\
3267(170) \\
60 \cdot 7(5 \cdot 0) \\
7 \cdot 3(0 \cdot 6)\end{array}$ & $\begin{array}{l}90 \\
(68-112) \\
300 \\
(180-420) \\
87 \cdot 5(4 \cdot 3) \\
246(18 \cdot 1) \\
66 \cdot 7(4 \cdot 1) \\
65 \cdot 7(3 \cdot 9) \\
97 \cdot 7(5 \cdot 8) \\
3120(221) \\
61 \cdot 0(5 \cdot 4) \\
3 \cdot 0(0 \cdot 30)\end{array}$ & $\begin{array}{l}270 \\
(200-600) \\
300 \\
(180-420) \\
230(4 \cdot 8) \\
356(18 \cdot 0) \\
152(16 \cdot 7) \\
145(11 \cdot 1) \\
261(12 \cdot 2) \\
3657(197) \\
69 \cdot 4(3 \cdot 7) \\
1 \cdot 6(0 \cdot 2)\end{array}$ & $\begin{array}{l}270 \\
(200-600) \\
3000 \\
(1800-4200) \\
265(11 \cdot 7) \\
1919(10 \cdot 4) \\
153 \cdot 6(9 \cdot 7) \\
180 \cdot 6(9 \cdot 8) \\
299(17 \cdot 9) \\
3716(237) \\
70 \cdot 5(3 \cdot 6) \\
1.3(0 \cdot 1)\end{array}$ \\
\hline
\end{tabular}

a Data calculated by expressing total inspired volumes as percentages of each individual's FVC, measured before aerosol administration. 
(100 seconds) were recorded using a gamma camera (GE Maxicamera 400A) fitted with a high resolution, low energy, parallel hole collimator and coupled to a SUN SPARC station IPX computer operating with commercial software (MAPS 10000; Link Medical Systems, Bourne End, UK). A complete record of each subject's inspiratory flow before, during, and after dosing was downloaded in real time from the SmartMist device to a personal computer running software developed for this purpose.

On a single occasion before the randomised study each volunteer received an $81 \mathrm{~m}$-krypton ventilation scan (anterior and posterior images) by tidal breathing ${ }^{81 \mathrm{~m}} \mathrm{Kr}$ gas from a generator (Regional Radioisotope Centre, Dudley Road Hospital, Birmingham, UK) via a mouthpiece until views containing 200000 counts were accumulated (100-200 seconds) by the gamma camera fitted with a medium energy collimator. A region of interest was drawn around the $20 \%$ contour of the geometric mean ventilation image to delineate the lung margin of each volunteer. This was superimposed on the anterior and reflected posterior deposition images, and a second region of interest drawn around an area equivalent to the peripheral lung using an established method. ${ }^{8}$ The ratio between aerosol counts in the central and peripheral zones $(\mathrm{C} / \mathrm{P})$ was normalised with respect to regional lung volume by division of this value by a $\mathrm{C} / \mathrm{P}$ ratio obtained from the corresponding geometric mean ventilation image. The resultant parameter is the regional aerosol distribution per unit volume, specific $\mathrm{C} / \mathrm{P}(\mathrm{sC} / \mathrm{P})$.

Regions of interest were also drawn to encompass radioactivity located in the stomach and mediastinum with a further area selected to represent background radiation. After back-

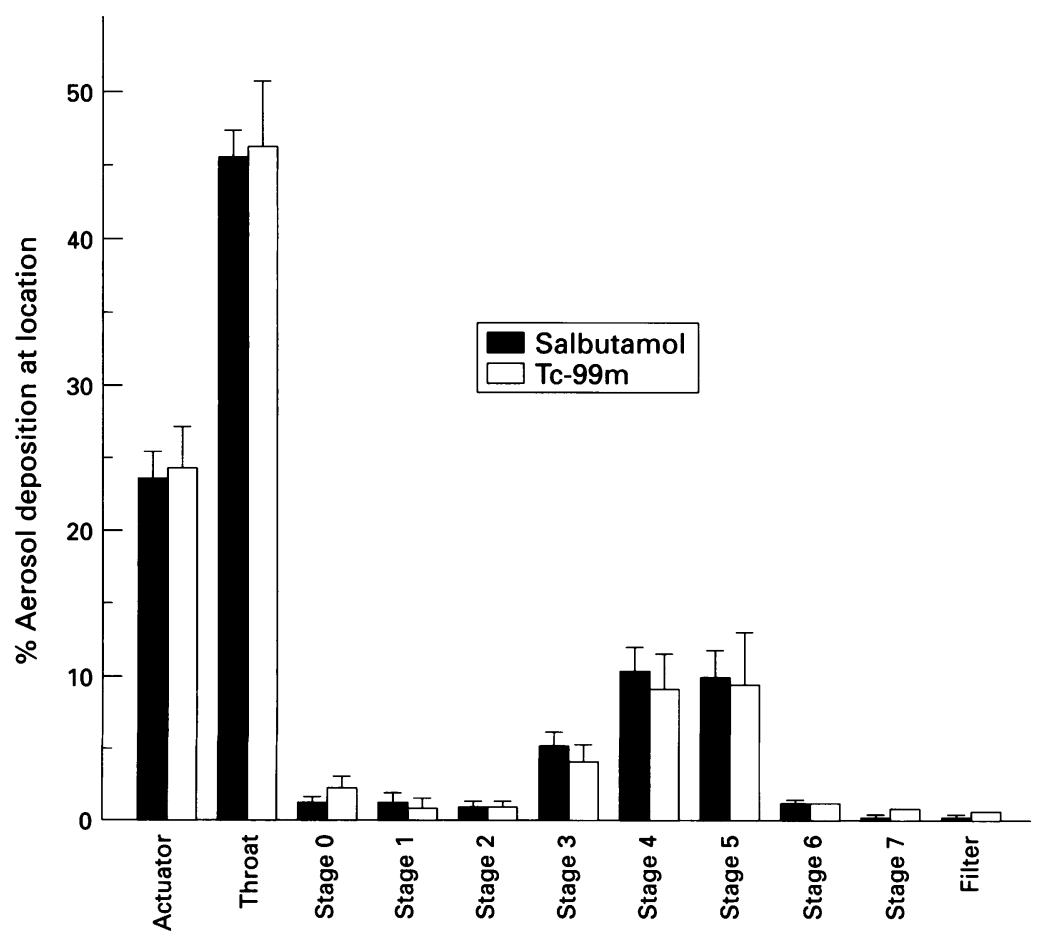

Figure 2 Histogram of the relative deposition of salbutamol and ${ }^{99 m} T c$ after actuation of radiolabelled salbutamol MDIs into an Andersen eight stage cascade impactor. Effective cut off diameters (at a flow rate of $28 \cdot 3 \mathrm{l} / \mathrm{min}$ ) for stages $0-7$ are $9 \cdot 0,5 \cdot 8,4 \cdot 7,3 \cdot 3,2 \cdot 1$, $1 \cdot 1,0 \cdot 7$, and $0 \cdot 4 \mu \mathrm{m}$, respectively. ground correction the geometric mean counts in those areas of interest were calculated from the appropriate pair of anterior and posterior counts. Background correct mouth and pharyngeal counts were determined from the lateral views, while the radioactivity remaining in the actuator or deposited on the filter was determined by placing these items directly on the collimator of the gamma camera for a 30 second acquisition. The relative distribution of radioactivity in each of the areas of interest was calculated after further correction of counts in the anatomical regions for attenuation. For the lungs and stomach/mediastinum this was determined by measuring transmittance of radioactivity from a ${ }^{99 \mathrm{~m}} \mathrm{Tc}$ flood source through the thorax of each volunteer, and comparison with a calibration curve of transmittance through acrylic polymer (Perspex) shields $(1-25 \mathrm{~cm}$ thickness) to yield an "equivalent Perspex thickness". The reciprocal of transmittance through half this thickness of Perspex gave a value for attenuation of activity in each region. Attenuation in the mouth and pharynx was calculated from the anatomical dimensions of these structures.

\section{STATISTICAL ANALYSIS}

Comparisons between groups were made using two way ANOVA using a 5\% level for type I errors. Where ANOVA indicated significant differences and more than two groups were involved in the experiment, Duncan's new multiple range test was used to identify significant differences between specific groups. A p value of less than 0.05 was considered significant.

\section{Results}

The deposition data of emitted aerosol droplets fired into the Andersen sampler from the ${ }^{99 \mathrm{~m}} \mathrm{Tc}$-labelled salbutamol MDIs are shown in fig 2 . In accordance with previously published data there was no significant mismatch in the fractionation of the aerosol components, indicating the ability of ${ }^{99 \mathrm{~m}} \mathrm{Tc}$ to follow the distribution of the salbutamol drug particles following oral inhalation. The mean (SD) values $(n=3)$ for respirable fraction (\% emitted dose $<5.8 \mu \mathrm{m}$ ) were therefore equivalent for salbutamol $(28.54(2.92) \%)$ and the radiotracer $(26.69(5.51) \%)$. The Andersen impactor, having eight collection stages with cutoff diameters ranging from $9.0 \mu \mathrm{m}$ down to $0.4 \mu \mathrm{m}$, represents a rigorous in vitro test because it is able to scrutinise potential differences between the aerosol components in this respirable range. ${ }^{5}$ The mean mass median aerodynamic diameters for salbutamol and ${ }^{99 \mathrm{~m}} \mathrm{Tc}$ were also statistically equivalent $(2 \cdot 60$ $(0.19) \mu \mathrm{m}$ and $2.76(0.46) \mu \mathrm{m}$, respectively) as were the calculated geometric standard deviations $(1.64(0.09)$ and $1.78(0.05)$, respectively).

Mean shot potencies (expressed as a percentage of expected output per valve actuation) at the start and end of pack shot life were also highly consistent: for shots $9 / 10$ the respective amounts of salbutamol and ${ }^{99 \mathrm{~m}} \mathrm{Tc}$ determined 


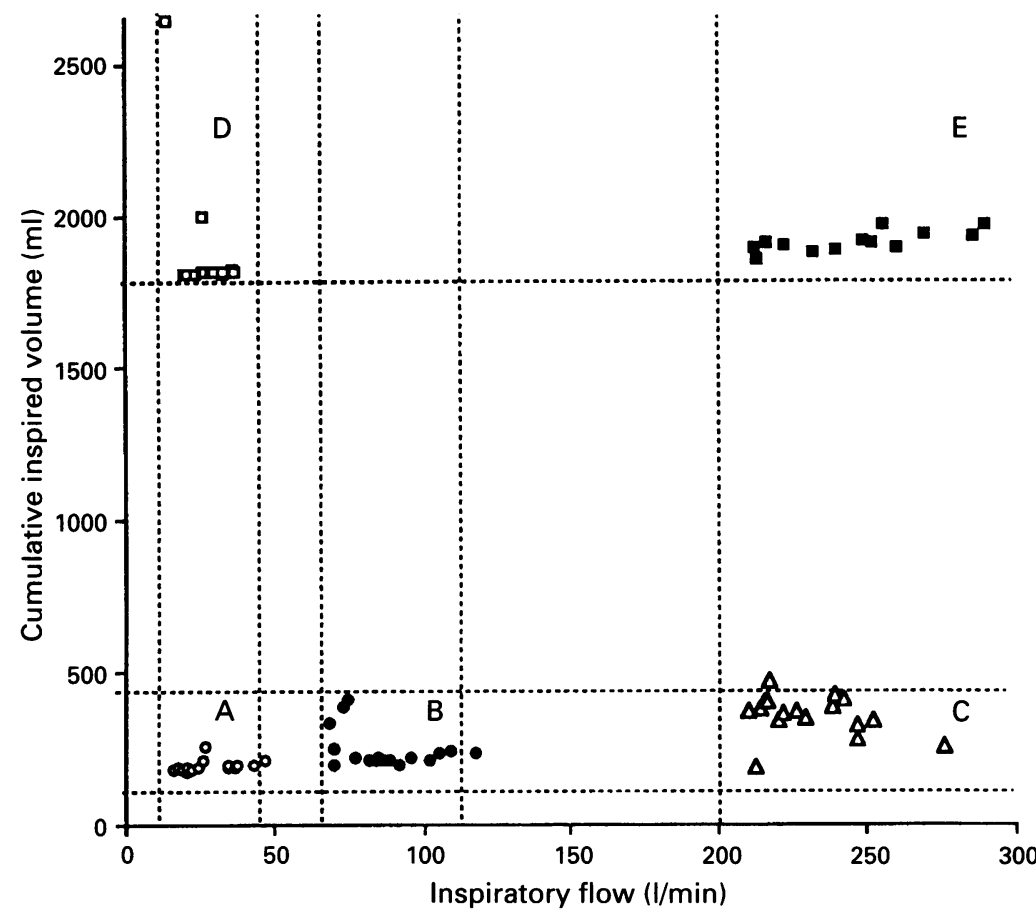

Figure 3 Individual firing points recorded by the SmartMist device during the aerosol deposition study. Each firing point is defined in terms of inspiratory flow and cumulative inspired volume. Symbols refer to firing points programmed to fire slowlearly (O), medium/early (O), fast/early $(\triangle)$, slow/late $(\square)$, and fast/late ( $)$. Numerical descriptions of these firing points are listed in table 1. The broken lines depict lower and upper limits set for the inspiratory flow and cumulative inspired volume. For reasons of clarity the upper limit of cumulative inspired volume for the "late" settings and the upper limit of inspiratory flow for the "fast" settings are not shown. Areas bordered by the limit lines represent acceptable actuations described by $(A)$ the slowlearly, (B) medium/early, (C) fastlearly, (D) slow/late, and (E) fast/late settings.

per actuation were $103 \cdot 7(6 \cdot 17) \%$ and $97 \cdot 7$ $(6 \cdot 0) \%$ of expected valve delivery $(100 \mu \mathrm{g}$ salbutamol, $5 \mathrm{MBq}{ }^{99 \mathrm{~m}} \mathrm{Tc}$ ), and for shots $35 / 36$ the respective values were $101 \cdot 2(10 \cdot 6) \%$ and $104.8(8 \cdot 66) \%$. These data provide further evidence of the homogeneity of dispersion of salbutamol and ${ }^{99 \mathrm{~m}} \mathrm{Tc}$ in the radiolabelled suspension formulation.

Table 2 Fractional deposition of ${ }^{99 m}$ Tc-labelled salbutamol pressurised aerosols following inhalation from a SmartMist device $(n=9)$

\begin{tabular}{|c|c|c|c|}
\hline \multirow{2}{*}{$\frac{\text { Firing point }}{\text { Slow/early }}$} & \multirow{6}{*}{$\begin{array}{l}\text { Location } \\
\text { Actuator } \\
\text { Oropharynx } x^{\mathrm{a}} \\
\text { Lungs } \\
\text { Peripheral lung } \\
\text { Exhaled }\end{array}$} & \multicolumn{2}{|c|}{$\begin{array}{l}\text { Mean (SE) deposition } \\
(\%) \text { at location }\end{array}$} \\
\hline & & $21 \cdot 0$ & $(1 \cdot 6)$ \\
\hline & & $65 \cdot 0$ & $(3 \cdot 0)$ \\
\hline & & $14 \cdot 1$ & $(2 \cdot 0)$ \\
\hline & & $7 \cdot 5$ & $(1 \cdot 1)$ \\
\hline & & 0.5 & $(0 \cdot 1)$ \\
\hline \multirow[t]{5}{*}{ Slow/late } & Actuator & $31 \cdot 5$ & $(1 \cdot 6)$ \\
\hline & Oropharynx & $57 \cdot 9$ & $(2 \cdot 0)$ \\
\hline & Lungs & $10 \cdot 6$ & $(1 \cdot 4)$ \\
\hline & Peripheral lung & $5 \cdot 6$ & $(0 \cdot 7)$ \\
\hline & Exhaled & 1.0 & $(0 \cdot 2)$ \\
\hline \multirow[t]{5}{*}{ Medium/early } & Actuator & $17 \cdot 6$ & $(2 \cdot 1)$ \\
\hline & Oropharynx & $63 \cdot 8$ & $(2 \cdot 5)$ \\
\hline & Lungs & $18 \cdot 6$ & $(1 \cdot 5)$ \\
\hline & Peripheral lung & $9 \cdot 1$ & $(1 \cdot 0)$ \\
\hline & Exhaled & 0.4 & $(0 \cdot 1)$ \\
\hline \multirow[t]{5}{*}{ Fast/early } & Actuator & $17 \cdot 7$ & (2.6) \\
\hline & Oropharynx & $73 \cdot 8$ & $(2 \cdot 4)$ \\
\hline & Lungs & $8 \cdot 4$ & $(0 \cdot 6)$ \\
\hline & Peripheral lung & $4 \cdot 2$ & $(0.6)$ \\
\hline & Exhaled & 0.6 & $(0 \cdot 2)$ \\
\hline \multirow[t]{5}{*}{ Fast/late } & Actuator & $21 \cdot 8$ & $(1 \cdot 8)$ \\
\hline & Oropharynx & $70 \cdot 6$ & $(2 \cdot 5)$ \\
\hline & Lungs & $7 \cdot 6$ & $(1 \cdot 0)$ \\
\hline & Peripheral lung & $3 \cdot 6$ & $(0 \cdot 4)$ \\
\hline & Exhaled & $1 \cdot 1$ & $(0 \cdot 4)$ \\
\hline
\end{tabular}

a Oropharyngeal deposition represents the totalled percentage deposition in the mouth, pharynx, and stomach/mediastinum.
b Percentage dose located within the region of interest defining peripheral lung deposition drawn according to Bennett et al. ${ }^{8}$
A graph of the firing points for all recorded actuations of the radiolabelled MDI (two actuations per volunteer per phase) obtained during the complete study is shown in fig 3 . In rare cases the actual firing point deviated from the programmed ranges for inspiratory flow or cumulative inspired volume. These were always minor and occurred for: (1) firing flow for one actuation programmed to fire at the slow/early setting ( $47 \mathrm{l} / \mathrm{min}$ compared with $45 \mathrm{l} / \mathrm{min}$ ) and one at the medium/early setting $(117 \mathrm{l} / \mathrm{min}$ compared with $112 \mathrm{l} / \mathrm{min}$ ); and (2) firing volume for a single fast/early setting $(472 \mathrm{ml} \mathrm{com-}$ pared with $420 \mathrm{ml}$ ). For all programmed modes of aerosol actuation, however, the mean firing values for inspiratory flow and cumulative inspired volume were within the set ranges (table 1). Mean firing flow occurred within $0.67 \%$ of the anticipated firing flow for the most consistent setting (slow flow and early in cumulative inspired volume), the maximum deviation $(13.2 \%)$ occurring at firing conditions defined by a fast flow coincident with a late point in the inspiratory manoeuvre. Similar levels of variability between firing points were also demonstrated with respect to cumulative inspired volume, but it was apparent that the volunteers usually met the flow/volume firing criteria towards the lower limit of the set volume range.

A summary of the parameters obtained for the complete inspiratory manoeuvres with the SmartMist operating at the five different firing points is shown in table 1 . It can be seen that the mean flow after actuation of the MDI was remarkably consistent with the overall mean flow; this, in turn, was significantly lower than the actual firing flow in all cases except for the slow/late setting. There were also small differences in the total inspired volume (TIV) between settings; however, when TIV is expressed as a function of FVC only the slow/ early group was significantly different from the other settings. This is probably explained by the unusually long duration of inhalation necessary following the aerosol administration event.

The results of aerosol deposition in the nine volunteers following oral inhalation are listed in table 2. There were significant differences in deposition profiles between firing point settings. The medium/early setting (firing at medium flow and early in the cumulative inspired volume) resulted in the highest total lung deposition which was significantly different from the other settings studied. The slow/ early setting resulted in the second highest lung deposition followed by the slow/late setting which was equivalent to the fast/early setting. The fast/late setting resulted in the lowest value of lung deposition at less than half the value of the optimum inspiratory firing point. There was no significant difference between the medium/ early and slow/early settings with respect to the extent of aerosol penetration into the peripheral zone of the lungs; however, both these values were significantly greater than those achieved when the MDI was programmed to fire at the highest flow and/or late in the inspiratory manoeuvre.

The values for peripheral lung deposition at settings defined by high flow or late during 
Table 3 Mean (SE) regional deposition (sC/P) of lung deposited dose of ${ }^{99 m}$ Tc-labelled salbutamol aerosols emitted from the SmartMist device $(n=9)$

\begin{tabular}{ll}
\hline Firing point description & Mean $(S E)$ sC/P \\
\hline Slow/early & $1.35(0 \cdot 17)$ \\
Slow/late & $1.28(0 \cdot 15)$ \\
Medium/early & $1.51(0 \cdot 14)$ \\
Fast/early & $1.73(0.43)$ \\
Fast/late & $1.44(0 \cdot 20)$ \\
\hline
\end{tabular}

inspiration, however, merely reflect the smaller extent of total lung deposition as there was no significant difference between the computed values of $s C / P$ (table 3 ). For each setting the value for $\mathrm{s} C / \mathrm{P}$ was $>1$, reflecting a more central than peripheral deposition pattern. Oropharyngeal deposition (calculated by totalling the corrected counts in the mouth, pharynx, and stomach/mediastinum regions) was significantly promoted by a rapid flow rate which prevailed at either an early or late firing point during inhalation. The slow/late setting resulted in the lowest oropharyngeal deposition as a result of a significant increase in the actuator associated dose. With all other treatments aerosol deposition in the actuator was similar to values obtained in the in vitro experiments. These are somewhat higher than is normally obtained with the standard Ventolin actuator, probably because of the slightly narrower actuator orifice $(0.457 \mathrm{~mm}$ compared with $0.508 \mathrm{~mm}$ ) in combination with the more flattened geometry of the SmartMist mouthpiece. The exhaled portion of the inhaled aerosol was, in all cases, less than $1 \%$. Although there was an observed tendency for a higher proportion of dose to be exhaled following a late firing point during inspiration, there was no significant difference between any of the treatments studied.

\section{Discussion}

This study has shown the ability of the SmartMist device to automatically actuate an MDI canister at a preprogrammed point during inhalation, defined by both flow rate and cumulative inspiratory volume. In previous studies in which the effect of inhalation mode on lung deposition of aerosols administered from MDIs has been investigated it has been common for inhalation to be controlled by specially designed apparatus comprising an MDI actuator connected in line to a pneumotachograph. ${ }^{4-14}$ Using this system the cumulative inspiratory volume at which the canister is fired can be preset. Inspiratory flow can be controlled by using volunteers well trained in the appropriate inhalation technique ${ }^{414}$ or by creating a fall in pressure across the inlet port of the apparatus - for example, with small holes to restrict flow - such that the achievable flow rate is constrained by maximum inspiratory effort. ${ }^{121516}$ With the SmartMist device resistance to flow is identical to that through a conventional MDI actuator. Moreover, the SmartMist device only controls the moment of firing and measures but does not directly control post-delivery flow, although the latter is obviously influenced by the training period each volunteer receives. The volunteers in the present study were naive with respect to the use of MDIs but were competent in activating the SmartMist at the various settings after 6-10 practices with a placebo canister.

At present optimisation of drug delivery to the lung from MDIs is obtained by training individuals to use the correct inhaler technique, or by resorting to spacer devices ${ }^{17}$ or breath actuated systems (such as the Autohaler). ${ }^{4}$ Use of the SmartMist device may have advantages over these other systems in that it controls the firing event in terms of the inspiratory flow and cumulative inhaled volume; both these factors were shown to have an effect on lung deposition. Conventional breath actuated systems merely restrict aerosol actuation until the patient commences inspiration - that is, there is no precise control over the flow and volume at which actuation occurs. Spacers optimise aerosol delivery by providing a chamber for modifying the size and velocity characteristics of the inhaled aerosol cloud. Consequently, they are bulky compared with a standard MDI or one loaded into a SmartMist device.

One unexpected finding was the small but significant improvement in lung deposition which occurred after firing the MDI at a medium inspiratory flow and early in the cumulative inspired volume compared with firing at a slow/early inhalation point. Based on earlier gamma scintigraphic evidence, the optimum inhalation mode for use with MDIs is one involving a slow inhalation (about 301/ $\mathrm{min}$ ) and it is generally regarded that an inspiratory flow rate approaching $100 \mathrm{l} / \mathrm{min}$ is too fast. ${ }^{12}$ The reason for the conflicting data in this study is not known precisely, although it may be due to the fact that these current investigations were carried out in healthy volunteers rather than in patients with obstructive airways disease. Previously, however, no difference in total or regional deposition of pressurised aerosols has been found between normal and chronic bronchitic subjects. ${ }^{18} \mathrm{An}$ other possible explanation could be related to differences in the luminal dimension of the airways as a function of resistance to inspiration. For example, the airways may well be inflated to differing extents when slower rates of inhalation (for example, $30 \mathrm{l} / \mathrm{min}$ ) are achieved through a device offering negligible resistance to flow compared with one offering a significant resistance to inspiration.

One further reason may be explained with reference to table 1 where it is evident that, as the set firing flow is increased, the overall mean flow becomes significantly lower than the actual firing flow; this latter parameter significantly correlates with the observed maximum flow. From this observation it may be hypothesised that inhaled flow rates averaged over the entire inspiratory manoeuvre (that may take 5 seconds or more to complete for a slow inhalation) may not be a valid indicator of the flow rate at the precise point of aerosol actuation or during the period when the aerosol initially penetrates into the lung. In other words, a measured overall mean flow of $100 \mathrm{l} / \mathrm{min}$ may occur in a situation 
where the MDI is actually fired at a significantly higher flow rate. This would be especially true if the administration device did not include a restrictor to constrain the inhaled flow.

It has recently been shown ${ }^{16}$ that the large airway response to salbutamol emitted from an MDI connected to a large volume spacer device is improved when adopting a faster $(57 \mathrm{l} / \mathrm{min}$ ) rather than a slower $(25 \mathrm{l} / \mathrm{min})$ inspiratory manoeuvre, a finding supported by a greater central deposition of the inhaled dose at the higher flow rate. Significant changes in regional lung deposition have also been reported for other MDIs inhaled at different flow rates. ${ }^{14}$ While there were differences in the extent of peripheral and central lung deposition for the various firing points used in this study, these simply correlated with total lung deposition; no firing point dependence was demonstrated for $\mathrm{sC} / \mathrm{P}$.

In conclusion, the SmartMist device allowed reproducible actuation of an MDI at a preprogrammed point during inspiration. The extent of aerosol deposition in the lung was affected by a change in firing point and promoted by an inhaled flow rate of up to $90 \mathrm{l} /$ min - that is, the slow and medium setting used in these studies. In the healthy volunteers used in this study, the optimum conditions for aerosol lung deposition was an inhalation flow rate set between 68 and $112 \mathrm{l} / \mathrm{min}$ coincident with a firing point early during inspiration. The data suggest the importance of further investigations with this device in patients with obstructive lung disease. One of the advantages, however, of using the microprocessor controlled device is that aerosol administration becomes more controlled, a prerequisite for investigating the lung as a route of delivery of systemically active drugs. Under these circumstances the firing conditions described above could be used to improve the dose to lung and hence the bioavailable fraction of the inhaled drug.
AMR gratefully acknowledges the support of a research assistantship awarded by Aradigm Corporation.

1 Colthorpe P, Farr SJ, Taylor G, Smith IJ, Wyatt D. The pharmacokinetics of pulmonary delivered insulin: a compharmacokinetics of pulmonary delivered insulin: a comparison of intratracheal and aerosol
rabbit. Pharm Res 1992;9:764-8.

2 Newman SP. Scintigraphic assessment of therapeutic aerosols. Crit Rev Ther Drug Carrier Sys 1993;10:65-109.

3 Summers QA, Clark AR, Hollingworth A, Fleming J, Holgate ST. The preparation of a radiolabelled aerosol of nedocromil sodium for administration by metered dose inhaler that accurately preserves particle size distribution of the drug. Drug Invest 1990;2:90-8.

4 Newman SP, Weisz AWB, Talaee N, Clarke SW. Improvement of drug delivery with a breath actuated pressurised aerosol with poor technique. Thorax 1991;46: $712-6$

5 Clarke JG, Farr SJ, Wicks, SR. Technetium-99m labelling of suspension type pressurised metered dose inhalers comprising various drug/surfactant combinations. Int $\mathcal{f}$ Pharm 1992;80:R1-5.

6 Cyr TD, Graham SJ, Li KYR, Lovering EG. Low first spray content in albuterol metered-dose inhalers. Pharm Res 1991;8:658-60.

7 Farr SJ, Kellaway IW, Parry-Jones DR, Woolfrey SF. 99mtechnetium as a marker of liposomal deposition and clearance in the human lung. Int $\mathcal{f}$ Pharm 1985;26:303-16.

8 Bennett WD, Messina MS, Smaldone GC. Effect of exercise on deposition and subsequent retention of inhaled particles. F Appl Physiol 1985;59:1046-54.

9 Newman SP, Pavia D, Morén F, Sheahan NR, Clarke SW. Deposition of pressurised aerosols in the human respiratory tract. Thorax 1981;36:52-5.

10 Newman SP, Pavia D, Clarke SW. Improving the bronchial deposition of pressurised aerosols. Chest 1981;6:909-11.

11 Dolovich M, Ruffin RE, Roberts R, Newhouse MT Optima delivery of aerosols from metered dose inhalers. Chest delivery of aerosc.

12 Newman SP, Pavia D, Garland N, Clarke SW. Effects of various inhalation modes on the deposition of radioactive pressurised aerosols. Eur $₹$ Respir Dis 1982;63 (Suppl): $57-65$.

13 Newman SP, Clark AR, Talaee N, Clarke SW. Pressurised aerosol deposition in the human lung with and withou an "open" spacer device. Thorax 1989;44:706-10.

14 Newman SP, Clark AR, Talaee N, Clarke SW. Lung deposition of $5 \mathrm{mg}$ Intal from a pressurised metered dose inhaler assessed by radiotracer technique. Int $\mathcal{f}$ Pharm 1991;74:203-8.

15 Laube BL, Georgopoulos A, Adams GK. Preliminary study of the efficacy of insulin aerosol delivered by oral inhalation of the efficacy of insulin aerosol delivered by oral inhalation in diabetic patients. $\mathscr{F} A M A$ 1993;269:2106-9.

16 Laube BL, Creticos PS, Dalby RN, Norman PS. Optimizing the effect of inhaled medications by altering inspiratory flow rate. In: Byron PR, Dalby RN, Farr SJ, eds Respiratory drug delivery IV. Richmond: VCU, 1994:173-8.

17 Newman SP, Millar AB, Lennard-Jones TR, Morén F, Clarke SW. Improvement of pressurised aerosol deposition with Nebuhaler spacer device. Thorax 1984;39:935.

18 Spiro SG, Singh CA, Tolfree SEJ, Partridge MR, Shor MD. Direct labelling of ipratropium bromide in norma subjects and patients with chronic bronchitis. Thorax 1984 39:432-5. 